CAHIERS DE

NARRATOLOGIE

\section{Cahiers de Narratologie}

Analyse et théorie narratives

17 | 2009

Stéréotype et narration littéraire

\title{
Les stéréotypes lexicalisés comme répétitions connotées dans une fugue littéraire : Diálogo en Re Mayor de Javier Tomeo
}

Sylvie Fournié-Chaboche

\section{OpenEdition}

Journals

Édition électronique

URL : http://journals.openedition.org/narratologie/1250

DOI : 10.4000/narratologie. 1250

ISSN : 1765-307X

Éditeur

LIRCES

Référence électronique

Sylvie Fournié-Chaboche, «Les stéréotypes lexicalisés comme répétitions connotées dans une fugue littéraire : Diálogo en Re Mayor de Javier Tomeo », Cahiers de Narratologie [En ligne], 17 | 2009, mis en ligne le 22 décembre 2009, consulté le 04 mai 2019. URL : http://journals.openedition.org/ narratologie/1250 ; DOI : 10.4000/narratologie.1250

Ce document a été généré automatiquement le 4 mai 2019.

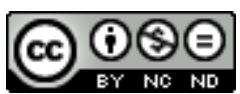

Cahiers de Narratologie - Analyse et théorie narratives est mis à disposition selon les termes de la licence Creative Commons Attribution - Pas d'Utilisation Commerciale - Pas de Modification 4.0 International. 


\title{
Les stéréotypes lexicalisés comme
} répétitions connotées dans une fugue littéraire : Diálogo en Re Mayor de Javier Tomeo

\author{
Sylvie Fournié-Chaboche
}

1 Le concept de stéréotype, de par son étymologie (référence à une production de formes figées dans le domaine de la typographie) invite à étudier le rapport qu'il entretient avec les formes fixes (au sens linguistique du terme). Or la lecture des textes théoriques sur le stéréotype, fait ressortir que si ces expressions figées sont systématiquement évoquées lors des analyses de la stéréotypie, elles sont également rapidement évacuées de ce champ d'étude. Ainsi Ruth Amossy et Anne Herschberg Pierrot consacrent deux petites pages aux locutions figées dans leur ouvrage Stéréotypes et clichés ${ }^{1}$ (1997) Ces auteurs précisent à juste titre que toutes les locutions ne sont pas des clichés car «la présence de figures stylistiques, prêtes à la remotivation sémantique en contexte, paraît nécessaire à la production de clichés» (p. 88) et que tous les clichés ne sont pas des locutions car ils n'ont pas forcément le même degré de figement que ces dernières. Ruth Amossy et Anne Herschberg Pierrot remarquent également que locutions et clichés n'appartiennent pas au même champ disciplinaire, les premières intéressant les linguistes pour des questions morphosyntaxiques et lexicales, alors que les seconds relèvent de la stylistique. Partant du postulat que la forme fixe dans le discours littéraire n'intéresse pas en tant que lexie mais en tant que forme lexicalisée, résultant d'un processus de répétition diachronique qu'elle connote, cet article propose précisément une étude stylistique des locutions et de la forme de stéréotypie qu'elles comportent, celle-ci devant-être définie.

2 L'analyse part du constat de l'usage abondant des stéréotypes lexicalisés que sont les formes fixes telles que les syntagmes figés (locutions) ou les énoncés figés (proverbes, dictons, sentences, etc.) dans l'œuvre littéraire de Javier Tomeo, écrivain espagnol contemporain. Pourquoi l'auteur utilise-t-il autant d'expressions figées? Ont-elles une 
fonction littéraire? L'idée de constante dans l'écriture d'un auteur est bien sûr indissociable de la notion de style. Dans l'art en général, la définition d'un style ne correspond-elle pas à la reconnaissance d'un ensemble de traits constants caractéristiques d'une époque, d'un genre? Il en est de même dans le domaine plus spécifiquement littéraire. Cette perception du style peut s'appliquer à un groupe d'œuvres mais aussi plus particulièrement à un auteur ${ }^{2}$. De ces deux acceptions, la seconde, qui a vu le jour au XIXème siècle ${ }^{3}$, est la plus délicate puisqu'elle offre le moins de points de repères. Il ne s'agit plus d'établir des liens entre une œuvre et une autre selon des critères culturellement acceptés. Décrire le style d'un auteur reviendrait à établir des liens au sein même de ses textes. Tzvetan Todorov définit le style comme «le choix que tout texte doit opérer parmi un certain nombre de disponibilités contenues dans la langue ${ }^{4}$.» Déterminer le style de Javier Tomeo consisterait donc à déceler les variables en langue qui seraient devenues des constantes dans son discours, à définir l'ensemble des traits formels caractéristiques de son écriture. La mission est vaste, voire impossible. On peut néanmoins, considérer que l'emploi d'expressions figées permet d'appréhender, ne serait-ce que très partiellement, le style de l'auteur même si d'aucuns considèreraient précisément la présence de stéréotypes lexicalisés (locutions, proverbes, citations, etc.), comme le négatif d'un style personnel.

3 Cela amène à poser le problème de l'originalité en littérature. Peut-on définir le style de Tomeo en faisant abstraction de ces emprunts et en s'intéressant uniquement à ce qui peut apparaitre comme de la pure création? La réponse est clairement négative. Il suffirait de retirer les proverbes de la prose de Javier Tomeo pour qu'elle perde de sa saveur. Les expressions et énoncés figés font non seulement partie intégrante du style littéraire de l'auteur mais ils constituent un élément-clef de la définition de celui-ci. Pourtant, comme l'a souligné Fernando Lázaro Carreter, folklore et littérature sont deux systèmes de production et de réception distincts. La différence fondamentale est précisément liée au fait que la littérature est mue par un désir d'originalité5. Comment le folklore peut-il alors contribuer à la littérarité d'un texte, laquelle ne semble pouvoir se définir que par l'originalité ? C'est du moins une conception moderne de la littérature. La présence au sein d'un texte de formules toutes faites n'était pas si paradoxale avant le XVIème siècle, époque où l'originalité commence à trouver place en littérature ${ }^{6}$. Auparavant, il était de bon goût de s'inspirer des prédécesseurs. L'autorité était nécessaire. Elle permettait un terrain d'entente avec le lecteur qui connaissait ces formules ou ces citations. Cela ne se faisait pas de parler de soi et l'autorité apparaissait un peu, de ce point de vue, comme la garante de la décence du discours. Selon Lázaro Carreter la littérature a fait une victime dans son désir d'affranchissement du carcan du folklore. Il s'agit du Refranero ${ }^{7}$, c'est-à-dire de l'ensemble des proverbes populaires. Pourtant, celui-ci est bien présent chez l'auteur contemporain Javier Tomeo. Or, si aujourd'hui le lieu commun en littérature est souvent de faire du neuf, nous posons l'hypothèse que l'originalité de cet écrivain consiste précisément à cultiver le « déjà dit » sous toutes ses formes.

4 Après avoir posé les problèmes théoriques du rapport entre stéréotypie et figement linguistique, et proposé le concept d'isotopie de la répétition, cet article analysera l'écriture répétitive de Diálogo en Re Mayor ${ }^{8}$ afin de mettre en lumière la contribution des stéréotypes lexicalisé à l'esthétique du roman.

5 Le figement linguistique donne lieu à différents résultats en langue : il y a d'une part les énoncés figés (proverbes, sentences, dictons, etc.) formant une parémie autonome ; il y a 
d'autre part les syntagmes figés formant des expressions phraséologiques à intégrer nécessairement dans le discours. Ces dernières, également appelées locutions, constitueront notre objet d'étude.

6 Au début du vingtième siècle, Charles Bally observe l'absence de correspondance systématique entre l'unité de pensée et le mot graphique et avance l'idée de «l'illusion du mot » lequel peut recouvrir plusieurs unités de pensée, de même qu'une seule unité de pensée peut s'étendre sur plusieurs mots9. Depuis, la linguistique a développé l'idée de signe minimal (morphème) et d'unité fonctionnelle (lexie). Le mot, limite inférieure du lexique, peut comprendre plusieurs morphèmes. De même, la lexie peut être constituée de plusieurs mots. Ce sera alors une lexie complexe définie par Bernard Pottier comme une "séquence en voie de lexicalisation à des degrés divers ${ }^{10}$ ». En tant qu'unité lexicale mémorisée, le syntagme que constitue la locution fait donc partie du lexique. Comme n'importe quel autre mot simple, la locution appartient au code de la langue. Elle est généralement idiomatique (le signifié global de l'expression ne correspond pas à la somme des signifiés des éléments composant le groupe). Elle est en revanche toujours figée. D'un point de vue formel, elle relève effectivement de la linguistique. Une des définitions les plus abouties du figement linguistique est celle proposée par George Misri :

Nous considérons donc comme figement tout groupe de monèmes qui présente un blocage total ou quasi-total des axes paradigmatique et syntagmatique, c'est-à-dire une impossibilité ou une réduction importante des possibilités de commutation et/ ou d'expansion partielle ${ }^{11}$.

7 Il convient donc d'étudier le rapport entre stéréotypie et figement linguistique. Dans son analyse très pointue du stéréotype, Jean-Louis Dufays (2004) explique que ce denier affecte trois niveaux de réalités : le langage, la structure linéaire et configurationnelle des genres discursifs et les représentations idéologiques. Il précise par ailleurs que la stéréotypie est dotée de sept caractéristiques :

- une caractéristique structurelle: le semi-figement des composants, qui sont comme quasi collés l'un à l'autre et au contenu qu'ils servent à désigner ;

- trois caractéristiques quantitatives : la fréquence d'emploi, le caractère collectif et le caractère durable ;

- un trait lié à la production du phénomène : son caractère «inoriginé » et donc anonyme ;

- deux traits liés à l'énonciation et à la réception du phénomène : le caractère automatique de son usage et son caractère axiologiquement problématique et réversible ${ }^{12}$.

Or, alors que dans ses écrits précédents les phénomènes langagiers concernés incluaient les expressions (« tourner la page ») ou énoncés ( «il n'y a pas de fumée sans feu ») figés ${ }^{13}$, la notion de figement disparait de son article de 2004 «Rumeur et stéréotypie » au profit de collocations non lexicalisées du type «bocage verdoyant». L'auteur reprend en revanche à Greimas la notion de semi-figement et s'en explique dans une note :

[...] le figement complet et définitif, [...] ôte au phénomène son caractère discutable et donc sa dimension axiologique. Le concept de semi-figement vient de Greimas (1960), qui a opportunément distingué sur cette base les syntagmes figés (comme battre pavillon, chercher midi à quatorze heures) des syntagmes semi-figés (comme verser des torrents de larmes, courir dans les frais bocages).

Plusieurs remarques s'imposent. Les représentations idéologiques peuvent être véhiculées par les expressions figées. Dufays donne l'exemple de la soi-disant bêtise des Belges. Pourquoi relèverait-elle plus du stéréotype que la force des Turcs qui, elle, est lexicalisée (et complètement figée) en français ? Si l'on ne peut définir le stéréotype par le 
figement, il n'est pas non plus envisageable d'exclure les expressions figées de ce champ d'étude car elles constituent un véritable vivier de stéréotypes en tous genres.

D'autre part, le figement est un phénomène qui affecte uniquement la forme de l'expression. Il n'intervient donc a priori en rien sur la dimension nécessairement axiologique du stéréotype. Pour Jean-Louis Dufays le stéréotype doit poser problème à au moins un des niveaux suivants :

- sur le plan informationnel et esthétique (si on se situe dans le cadre d'une esthétique moderne qui valorise l'effet de surprise), c'est un signe banal, prévisible, rebattu, usé et donc dénué de valeur ;

- sur le plan psychologique, c'est un signe intellectuellement faible, qui trahit le manque d'autonomie et de personnalité, et donc la dépendance, voire l'aliénation de son utilisateur ;

- sur le plan référentiel, c'est un signe simpliste, réducteur, trop général ou trop abstrait, voire carrément erroné, en tout cas inadéquat, qui trahit un déficit de concrétude, de précision et de complexité;

-sur le plan éthique (toujours s'il s'agit d'une représentation), c'est un signe injuste, discriminatoire, violent à l'égard des réalités et des personnes auxquelles on l'applique ${ }^{14}$.

11 Le carcan formel n'affecte en rien l'image et ce qu'elle véhicule. Certes, il impose une ou plusieurs combinatoires et en en excluant une ou plusieurs autres (on pourra être « fort comme un turc, fort comme un bœuf» mais pas "fort comme un sumo"), mais cette combinatoire imposée est tout aussi riche d'histoire, de représentations de connotations que si elle n'était pas figée. Elle l'est d'ailleurs peut-être plus puisqu'elle a fini par se figer. Même si « fort comme un turc » signifie très simplement 'très fort', l'image est bel et bien là et son emploi n'est pas neutre : il pose tout autant de problème au niveau référentiel que la bêtise des belges ou la lenteur des suisses. Une locution identique mais moins laudative telle que « saoul comme un polonais » pose en outre sérieusement problème au niveau éthique de par son caractère discriminatoire.

On ne peut certes pas réduire les locutions figurées (car seules celles-ci peuvent véhiculer un stéréotype) à cette unique catégorie de locutions adjectivales. Le stéréotype est peutêtre moins éclatant dans les locutions nominales, verbales, adverbiales car il ne sera pas souvent problématique d'un point de vue référentiel ou éthique, mais il le sera sans aucun doute d'un point de vue esthétique, notamment dans une œuvre littéraire. Des expressions comme «llevar agua a su molino » (apporter de l'eau à son moulin), «tener más razón que un santo » (avoir cent fois raison), « acabar como el rosario de la aurora » (finir en eau de boudin), «no parecer trigo limpio » (ne pas sembler très catholique ${ }^{15}$ ), pour ne donner qu'un échantillon de ce que recèle Diálogo en Re Mayor, ne sont-elles pas perçues comme des signes rebattus malgré leur appartenance au lexique ? Leur utilisation en littérature ne rajoute-t-elle pas à leur inconvenance alors qu'il était loisible à l'auteur de créer des métaphores inédites du discours libre pour dire la même chose? Au-delà de l'image véhiculée, le stéréotype n'est-il pas tout simplement mis en exergue par la perception de la forme figée et surtout du processus de répétition qui a contribué à ce figement? C'est la raison pour laquelle, du point de vue de la stéréotypie, il semble plus pertinent de considérer le processus de figement que son résultat plus ou moins arbitraire. D'où la notion de stéréotype lexicalisé et non pas seulement lexical.

La double constatation de la présence, dans l'œuvre de Javier Tomeo, d'un grand nombre d'expressions figées et de répétitions sous diverses formes amène à formuler une nouvelle série de questions. Y a-t-il un lien entre ces deux phénomènes? En d'autres 
termes, ceci revient à se demander s'il existe une quelconque relation entre la présence de ces formes fixes et le fait qu'elles apparaissent dans une œuvre construite sur le mode de la répétition. Si, comme on l'a évoqué, l'emploi de formules toutes faites a été, à une certaine époque, décrié en littérature au nom de l'originalité, c'est précisément pour lutter contre le sentiment de "déjà-vu » véhiculé par ce type d'expressions. Est-ce à dire que les locutions figurées et autres manifestations du savoir populaire constituent également une forme de répétition? Il s'agit, pour répondre à cette question, de bien analyser la nature de la répétition présente dans ces expressions.

Les expressions figées sont souvent issues de l'oralité mais peuvent également provenir de l'écriture et avoir connu, à un moment donné, un processus d'oralisation ${ }^{16}$ (transmission et lexicalisation par le biais de la répétition orale) ce qui n'est pas étranger à ce sentiment de " déjà-vu » ou plus exactement de " déjà-dit ». Ce dernier est inhérent à la présence même d'une forme fixe. Il suffit donc d'une seule occurrence de celle-ci pour que cette impression se produise. Il n'en est pas de même pour toutes les autres formes de répétitions que l'on peut trouver dans un texte. Dans ce dernier cas, ce sentiment est lié à la réitération, au sein d'un espace textuel défini, de mêmes phrases, de mêmes passages, de mêmes images, etc. Ces répétitions s'effectuent dans la synchronie du discours. Or pour Coseriu, il est précisément nécessaire de distinguer, dans la synchronie, la technique $d u$ discours du discours répété (les expressions et énoncés figés ${ }^{17}$ ). Cette appellation de discours répété est ambiguë mais, de ce fait, révélatrice de la complexité du problème. En effet, contrairement aux répétitions discursives (l'auteur se répète au fil de son texte), le discours répété de Coseriu ne désigne pas un phénomène de répétition concret (dont l'indice serait la multiplicité des occurrences de tel ou tel élément du texte), mais un phénomène linguistique issu d'un processus de répétition diachronique (qui a eu lieu dans le temps). La répétition ne figure pas nécessairement dans le texte lui-même mais en amont, car elle a contribué à la formation des expressions figées. Il y a, lors de l'emploi de toute forme figée, une rémanence de sa genèse puisque cette expression est le vestige de ce qui fut à l'origine, une création du discours libre qui, à force de répétitions et de mémorisations successives, a fini par se lexicaliser.

La répétition constitue donc bien un facteur commun aux deux phénomènes observés dans l'œuvre de Javier Tomeo, à savoir l'abondance de formes fixes d'une part et de répétitions diverses d'autre part. Mais le premier de ces deux phénomènes se distingue du second dans la mesure où ce "discours répété" a été accepté en langue et du fait que le sentiment de répétition ne découle pas nécessairement d'un nombre d'occurrences supérieur à un. Il a donc semblé nécessaire de lever l'ambigüité terminologique liée à la notion de " discours répété » d'Eugenio Coseriu, en faisant ressortir des caractéristiques propres à chacune de ces formes de répétitions.

16 Il y a d'une part les répétitions textuelles (intra-textuelles ou intertextuelles) qui correspondent aux réitérations discursives présentes au sein d'un texte écrit (ou d'un roman à un autre). Il y a d'autre part des répétitions connotées, c'est-à-dire les formes fixes, stéréotypes lexicalisés véhiculant une impression de déjà-dit. De par sa nature même, cette forme de répétition n'est pas opérationnelle dans une analyse thématique. Il conviendra de profiter des clefs qu'offre le structuralisme pour mettre au jour un système littéraire plus complexe, une structure plus profonde.

Les structures ne sont pas, à beaucoup près, des objets de rencontre, ce sont des systèmes de relations latents, conçus plutôt que perçus, que l'analyse construit à mesure qu'elle les dégage ${ }^{18}$. 
17 Le système de relations latent que l'on se propose de décrire ne repose pas principalement sur la sémantique. Ce qui y sera pertinent est bien moins la thématique (souvent anecdotique) de la réitération que la présence même de la réitération d'une réflexion ou anecdote tout au long d'un roman ou dans différents romans de Tomeo. De même, ce qui importe dans la présence des locutions figées est moins ce qu'elles dénotent ou les images qu'elles véhiculent, que le fait qu'elles soient issues d'un processus de répétition diachronique et que cette répétition soit connotée par leur présence même. Une isotopie étant formée, selon Greimas ${ }^{19}$, d'un réseau de récurrences relevant aussi bien de la connotation que de la dénotation, on peut alors poser l'existence d'une isotopie de la répétition qui regrouperait, au sein d'un même système, la répétition connotée par la présence de formes figées et les répétitions discursives contribuant aux diverses isotopies sémantiques. C'est seulement en passant par l'élaboration de ce système plus vaste qu'il est possible de relier le résultat en langue d'un phénomène de répétition (les expressions figées) à la répétition en discours qu'elle soit intra-textuelle ou intertextuelle (interne ou externe à l'œuvre). Ce système portant sur l'intégralité de l'œuvre de Javier Tomeo ayant déjà été mis en lumière ${ }^{20}$, on s'attachera, dans cet article, a présenter une analyse de détail du roman dans lequel le phénomène de répétition intra-textuelle est le plus abouti et également un de ceux qui comporte le plus de stéréotypes lexicalisés Diálogo en Re Mayor dans sa troisième et dernière version de 1993 (DRMc).

Diálogo en Re Mayor

Il convient à présent de dépasser la présentation théorique de l'isotopie de la répétition en mettant en lumière ses déclinaisons concrètes dans le roman Diálogo en Re Mayor. Il apparaît indispensable de donner un aperçu de l'écriture de Javier Tomeo pour montrer l'importance des stéréotypes lexicalisés de ce roman :

Aquellas palabras, como es lógico, "no me gustaron un pelo, pero hice de tripas corazón" y continué sonriendo "como si tal cosa". Le contesté que muy bien, que cada cual era libre de pensar lo que le diese la gana, pero que, a pesar de todo lo que él pudiese pensar, yo seguiría convencido de que los instrumentos de viento en general y los trombones en particular tenían sus virtudes y que si se tocaban "como Dios manda" podían producir en la orquesta efectos electrizantes.

- El sonido de los trombones - le dije, tratando de sostener su mirada de vampiro sin pestañear -, resulta siempre noble y majestuoso. Piense usted que fue Wagner, y no cualquier musiquillo "de tres al cuarto" quien los introdujo en las más modernas orquestas sinfónicas ${ }^{21}$ (p.12).

Il n'y a pas de pages qui ne comporte son lot de locutions figurées, parfois employées en cascade comme au début de l'exemple précédent.

Le roman présente également de nombreux proverbes comme «quien a los suyos se parece honra merece » (p. 20), « nunca es tarde cuando la dicha es buena » (p. 28), « el que tiene padrinos se bautiza » (p. 67), « contra gustos no hay disputas » (p. 72), «al César lo que es del César» (p. 76), des formules conversationnelles non proverbiales (c'est-à-dire sans contenu moralisateur) mais néanmoins lexicalisées comme " el mundo es pañuelo » (p. 65) ou encore des stéréotypes non lexicalisés comme "la prostitución es la profesión más antigüa del mundo" (p. 73). Comme pour les locutions ces différents types d'énoncés stéréotypés et plus ou moins figés peuvent s'enchaîner en cascade :

Dagoberto, mientras tanto, me fue dando varias definiciones del amor (...). Me dijo, entre otras cosas que el amor era un cocodrilo en el río del deseo, que los corazones enamorados nunca saben a dónde van, que el amor era ciego y que el enamorado y el pez, frescos habían de $\operatorname{ser}^{22}$ (p. 120). 
A côté de cette profusion de répétitions connotées (les stéréotypes lexicalisés), ce roman multiplie à loisir les répétitions intra-textuelles. L'écriture devant être considérée comme un « usage singulier du langage selon des normes (conscientes ou inconscientes) internes à l'œuvre considérée ${ }^{23}$ ", il est légitime de poser l'hypothèse que la norme de l'écriture de Diálogo en Re Mayor constitue un système dense de répétitions. Or, décrire un système littéraire revient à analyser ses constantes et les rapports entre elles. Il ne s'agira pas de décrire dans le détail et isolément chaque répétition, mais d'essayer, au contraire, de schématiser leur organisation au sein du texte.

Diálogo en Re Mayor est le récit de la conversation des deux seuls occupants d'un train. Le roman commence au moment où le personnage, Juan, prend un billet au guichet de la gare de départ et s'achève peu avant l'arrivée du train à la gare de destination quand le personnage saute du train. L'originalité de la narration provient du fait qu'au sein du déroulement diégétique unique, les personnages vont être amenés à rejouer, à plusieurs reprises, leur propre histoire, c'est-à-dire leur passé immédiat. Après un début de conversation tournant au cauchemar pour Juan, son tyrannique interlocuteur, Dagoberto, propose de reprendre la conversation à zéro, au moment où Juan est entré dans le compartiment :

- ¡Ah, sí, probemos otra vez! - gritó (...). ¡Qué pretensión la nuestra! ¿Cómo íbamos a entendernos al primer intento? ¡Intentémoslo otra vez! ¡Hemos empezado un crucigrama y no podemos dejar la mitad de las casillas en blanco !

- ¿Qué es lo que quiere usted volver a empezar? - le pregunté.

- Nuestro diálogo - respondió -. Volvamos a intentarlo ${ }^{24}$ (p. 63).

A peine Juan se représente-t-il, que Dagoberto suggère à nouveau de recommencer sur de meilleures bases et l'invite même à se lever pour pouvoir rejouer parfaitement la scène de son entrée dans le compartiment et du moment où il s'est assis :

- Amigo Juan - me propuso Dagoberto -, esta vez, para que las cosas salgan mejor, nos concederemos algunas ventajas. Vamos a partir de cero, sin prejuicios ni ideas preconcebidas. Imagínese usted que yo soy un hombre normal, dispuesto a compartir de buen grado el departamento con otro viajero. Salga otra vez al pasillo, vuelva a entrar y siéntese otra vez en el mismo sitio donde está ahora ${ }^{25}$ (DRMc63).

La conversation démarre à nouveau. Juan se présente comme joueur de trombone dans la fanfare municipale de la ville de K. Dagoberto demande à son interlocuteur s'il n'est pas commis voyageur, variant ainsi par rapport à la fois précédente où Juan avait anticipé la question. Mais le dialogue permet d'aborder également de nouveaux thèmes jusqu'à ce que Dagoberto décide une fois de plus de reprendre à zéro, l'invitant à se présenter à nouveau :

- Creo que es mejor que vuelva usted a presentarse-me pidió.

- Pues muy bien -le dije, armándome de paciencia ${ }^{26}$ (DRMc75).

Cette troisième reprise donne lieu à de nombreuses répétitions et variations sur les propos émis lors du précédent essai de conversation jusqu'à un nouveau désir d'interruption et de recommencement qui apportera également son lot de répétitions et de variations :

(...) me dijo que habíamos vuelto a fracasar y que debíamos intentar el diálogo una vez más.

- Es mejor que no perdamos el tiempo -me pidió-. Dígame otra vez cómo se llama, a qué se dedica y cuál es su destino ${ }^{27}$ (DRMc90).

Il convient de préciser que malgré le caractère anaphorique de ces dialogues, débutant généralement par le rituel de la présentation de Juan, la conversation qui s'ensuit peut 
prendre, dans chacun des cas, des tournures différentes. Si toutes comprennent des éléments répétés, chacune en offre une sélection, un ordre d'apparition ou une fréquence différentes, sans compter les innombrables variations inhérentes aux répétitions. Par exemple, la devinette sur la réaction d'un sultan à la vue d'un trombone à coulisse (et qui croyait que le musicien avalait et recrachait alternativement une partie de l'instrument), n'apparaît pour la première fois que lors de la deuxième reprise du dialogue :

¿Sabe, por cierto, lo que pensó un sultán la primera vez que vio un trombón de varas? Agárrese, porque la cosa tiene gracia: aquel servidor de Alá pensó que el músico se tragaba y devolvía alternativamente parte del instrumento.

¿No le parece gracioso ${ }^{28}$ ? (p. 68).

La deuxième occurrence a lieu lors de la troisième reprise dans des circonstances très similaires à celles de la fois précédente, suite à la présentation de Juan comme joueur de trombone à la fanfare municipale de la ville de J. Cette fois-ci l'anecdote du sultan est racontée directement sans le subterfuge de la devinette :

Luego empezó a contarme otra vez la historia del sultán que la primera vez que vio un trombón de varas pensó que el músico se tragaba y devolvía alternativamente el instrumento $^{29}$ (DRMc76).

Les troisième, quatrième, cinquième et sixième occurrences font partie de la quatrième et dernière reprise du dialogue initial. La première d'entre elles relève du même phénomène que les précédentes : elle est liée au nouveau départ de la conversation. L'originalité et l'ironie de cette variation réside dans la mauvaise foi de Dagoberto. Celui-ci feint en effet d'ignorer la situation qui l'amène à se répéter et dont il est responsable. Il joue ainsi complètement le jeu de la conversation reprise à zéro en faisant table rase de ce qui a déjà été dit, allant même jusqu'à s'étonner de ce que son interlocuteur connaisse déjà cette histoire. Il prend un malin plaisir à lasser son interlocuteur en ressassant la même histoire tout en feignant de la raconter pour la première fois. Juan ayant accepté le jeu se trouve donc contraint de l'écouter :

Me preguntó a continuación si conocía la historia del sultán, le dije que sí y quiso saber quién me la había contado.

- Me la contó usted mismo, hace un momento - le recordé.

- ¿Cómo puede ser eso posible - exclamó, enarcando las cejas -, si usted y yo acabamos de conocernos ${ }^{30}$ ? (DRMc91).

Les trois dernières apparitions de l'histoire du sultan ne sont pas imputables au jeu consistant à reprendre le dialogue à son début puisqu'elles ont lieu au cours de la même dernière tentative de conversation que l'exemple précédent. Il s'agit donc d'une nouvelle modalité de la répétition, relevant véritablement du ressassement. Alors que, cette fois-ci, Juan aurait toute légitimité à faire remarquer à son interlocuteur que celui-ci lui a déjà raconté l'histoire, il se résigne à l'entendre à nouveau, certainement pour ne pas froisser Dagoberto, faisant ainsi apparaître nettement le rapport de domination exercé par ce dernier sur l'autre voyageur. Dans l'exemple suivant, le personnage narrateur Juan se fait, par ailleurs, mentalement la remarque de la présence d'une variation dans l'histoire racontée puisque cette fois ci le sultan pense que le trombone à coulisse est comestible :

¿Y sabe usted lo que pensó un sultán la primera vez que vio un trombón de varas?

En aquella ocasión puse cara de sorpresa y negué con solemnes movimientos de cabeza para darle el gusto de que pudiese contarme otra vez la historia.

- Aquel sultán - me dijo, introduciendo una ligera variación respecto a la versión anterior-, pensó que los trombones de varas eran comestibles ${ }^{31}$ (p. 98). 
L'histoire n'est pas toujours détaillée dans le roman. La répétition consiste parfois simplement en la mention du récit effectué par Dagoberto. Cela n'enlève rien à l'impression de martèlement produite par ces reprises intervenant de surcroit dans un intervalle très restreint :

- Puestos a contar chistes cortos (...) yo conozco el del sultán y el trombón de varas. ¿Lo conoce usted?

Le dije nuevamente que no y mientras me lo contaba por enésima vez el tren se puso en marcha ${ }^{32}$ (p. 101).

Dagoberto continuó con su papel de abogado del diablo y una vez más sacó a relucir la historia del sultán.

- ¿Cree usted que hay muchos instrumentos musicales que puedan confundirse con un caramelo con mango ${ }^{33}$ ? (pp.106-107).

31 La répétition de l'histoire du sultan constitue un exemple parmi beaucoup d'autres du phénomène répétitif se produisant dans Diálogo en Re Mayor. L'analyse de cette récurrence a permis de faire apparaitre deux modalités de répétitions. Certaines sont liées au jeu consistant à recommencer plusieurs fois la conversation du début. D'autres, comme les dernières étudiées, sont purement gratuites et ne répondent à aucune logique si ce n'est à la volonté de Dagoberto de rendre fou son interlocuteur. Dans les deux cas, l'histoire du sultan correspond à une anecdote extérieure au cadre spatio-temporel de ce voyage en train dans lequel elle se trouve relatée à six reprises. Le terme d'anecdote a son importance car il confère un caractère unique à l'histoire en question. A un événement unique, correspondent donc des récits multiples. Pour Gérard Genette qui s'intéresse aux relations de fréquence entre récit et diégèse, il s'agit d'un cas de récit répétitif ${ }^{34}$ qu'il $^{\prime}$ résume par la formule suivante : «Raconter $\mathrm{n}$ fois ce qui s'est passé une fois ». Partant de la constatation que l'événement comme l'énoncé narratif ont la possibilité de se produire et de se reproduire, il identifie trois autres cas : «Raconter une fois ce qui s'est passé une fois », « Raconter $\mathrm{n}$ fois ce qui s'est passé $\mathrm{n}$ fois » et « Raconter une fois ce qui s'est passé $\mathrm{n}$ fois ». Le dernier ne se présente pas dans le roman. Ce n'est pas le cas pour les deux autres que Gérard Genette réunit sous le nom de récits singulatifs se caractérisant par un rapport d'égalité entre le nombre d'occurrences et le nombre d'événements. Si ce nombre est égal à un, la narration est tout à fait classique et il n'y a, bien sûr, pas de récurrences. $\mathrm{Au}$ delà, il y a répétition mais d'un autre type que celle étudiée avec l'exemple de l'histoire du sultan. Chaque fois que Juan se présente, chaque question posée sur le concours de passacailles ou sur la préférence entre montagne du nord ou du sud constitue autant de micro-événements mentionnés dans le récit autant de fois qu'ils apparaissent dans l'histoire. La nature des répétitions présentes dans Diálogo en Re Mayor est donc variée puisque ce roman combine récit singulatif et récit répétitif et qu'au sein de chacun de ces types de fréquences narratives la récurrence peut relever soit de la structure anaphorique de la diégèse (reprises du dialogue à son début), soit d'une attitude obsessionnelle des personnages.

On se propose à présent de représenter graphiquement ce maillage de récurrences tout au long du cheminement diégétique (Figure 1, en annexe en bas de page). Pour ce faire, il a fallu sélectionner quelques exemples de répétitions. On a fait le choix d'éliminer celles qui ne sont jamais proférées oralement par les personnages (par exemple les multiples constatations de Juan sur la couleur rouge des yeux de son interlocuteur). Ce choix s'est fait au profit de phénomènes plus atypiques du fait même que les récurrences interviennent dans un dialogue, ce qui lui confère un caractère grotesque ou absurde. 
Malgré cette sélection, les exemples intervenant dans cette schématisation ne constituent qu'un échantillon et ne prétendent pas à l'exhaustivité. Ils permettent néanmoins d'observer la structure répétitive du texte

L'examen de la densité globale des points sur le graphique donne une idée de l'ampleur du phénomène de répétition. Leur inégale densité dans le détail est également révélatrice. On navigue ainsi entre une longue plage de "répit" (seules trois récurrences différentes sont observées entre les pages 24 et 63) et des espaces textuels martelés, voire saturés par la répétition (par exemple page 9 à 24, 66 à 86, 91 à 113). Or ces concentrations sont symptomatiques de chaque début de dialogue. La distance entre les points d'une même couleur, donc sur un axe horizontal, montre de surcroît la nature de la diffusion d'une même répétition au cours du roman. En revanche, les oscillations de la courbe de répétition n'ont une signification que relative puisque les graduations de l'axe vertical du graphique figurent simplement l'ordre chronologique d'apparition de la première occurrence de chaque répétition. Plus la courbe monte, plus la répétition en question est apparue tardivement dans le texte et inversement. Ces oscillations permettent néanmoins d'apprécier l'organisation de ce que Roland Barthes appelle « un continu fugué, dans lequel des fragments identifiables rentrent sans cesse dans la course ${ }^{35}$ ». La comparaison musicale est intéressante, car la structure de Dialogo en Re Mayor est effectivement très semblable à celle d'une fugue avec l'entrée successive des voix (représentées sur l'axe vertical) et la répétition et variation sur un thème (représenté sur l'axe horizontal). En outre, l'enchevêtrement entre les répétitions concernant la préférence entre les trombones et les violons (apparu dès le dialogue initial) et celles concernant la préférence entre les montagnes du nord et celles du sud, n'ayant fait son entrée que lors de la deuxième reprise semble correspondre tout à fait au principe de poursuite caractérisant ce genre musical :

«En musique, ce terme [fugue] se réfère à une composition dans laquelle trois voix (ou plus) entrent en imitation l'une après l'autre, chacune pourchassant la précédente qui prend la "fuite" devant elle ${ }^{36} »$.

Il ne semble pas que l'on se soit jusqu'à présent beaucoup interrogé sur le titre de ce roman comme s'il était évident que la référence musicale procédait de la discussion sur les trombones et les violons. A la lumière de cette analyse sur la structure répétitive du roman, le titre prend tout son sens. Il se réfère moins au contenu du texte qu'à sa structure répétitive. Il n'est pas anodin d'attribuer au premier mot du titre Dialogue le qualificatif que l'on appliquerait à une composition musicale et, entre autre, à une fugue (dont la plus célèbre se trouve être en ré... mineur ${ }^{37}$ !) Ce dialogue est construit comme une fugue. Il en a l'organisation répétitive se caractérisant par la répétition sur le mode de la variation et de la translation. C'est encore à Roland Barthes qu'on emprunte ce terme :

«La rhétorique classique a donné sa réponse magistrale pendant des siècles, en édifiant une esthétique de la variation (dont l'idée de "développement" n'est que le mythe grossier); mais il y a une autre rhétorique possible, celle de la translation: moderne, sans doute, puisqu'on ne la trouve que dans quelques œuvres d'avantgarde ; et cependant, ailleurs, combien ancienne: tout récit mythique, selon l'hypothèse de Claude Lévi-Strauss, n'est-il pas produit par une mobilisation d'unités récurrentes, de séries autonomes (diraient les musiciens), dont les déplacements infiniment possibles, assurent à l'œuvre la responsabilité de son choix, c'est-à-dire sa singularité, c'est-à-dire son sens ${ }^{38}$ ? ». 

esthétique de la répétition comme principe d'écriture était déjà en germe dans le titre. Encore fallait-il en résoudre l'énigme. A moins qu'en changeant d'échelle on ne considère plutôt que Diálogo en Re Mayor n'est finalement qu'une voix de l'immense fugue littéraire que constitue l'œuvre de Javier Tomeo, tant les romans s'interpénètrent et se font écho, mais avec toujours la même constante: une écriture où abondent les stéréotypes lexicalisés.

A la différence de ce qu'écrit R. Barthes à propos de Mobile de Michel Butor, Diálogo en Re Mayor combine la variation et la translation dans une structure répétitive s'apparentant à une composition musicale : la fugue.

Diálogo en Re Mayor constitue la manifestation la plus aboutie de l'écriture répétitive de Javier Tomeo. La répétition est tellement constante qu'elle devient la norme de l'écriture de ce roman et pas seulement du discours musical comme il est précisé au tout début du roman. ("Richard Wagner había sido quien introdujo el leitmotiv como elemento cohesionador del discurso musical y como definidor de las situaciones dramáticas ${ }^{39 ")}$. A moins que ces deux discours ne se confondent... En effet, l'analyse de l'agencement de ces récurrences (translations et variations) a fait ressortir des similitudes avec certaines compositions musicales (la fugue en particulier). L'analogie entre la structure répétitive de ce dialogue et celle de la fugue donne au titre du roman Diálogo en Re Mayor tout son sens. On a montré à quel point les récurrences (sous des formes variées) innervent tout le texte.

Ces répétitions intra-textuelles trouvent un écho dans le style même de l'auteur. Ce dernier use et abuse de syntagmes et d'énoncés figés connotant, par leur présence même, le processus de répétition qui a fait de ce qui était à l'origine des éléments du discours libre, des stéréotypes lexicalisés. Employer une expression figée et figurée signifie en effet bien plus que la combinaison des dénotations liées au signifié idiomatique de l'expression et des connotations liées à l'image véhiculée. C'est aussi un acte symbolique par lequel on constitue, l'espace d'un instant, le dernier maillon d'une chaîne de locuteurs qui se sont appropriés, parfois depuis des siècles la même image devenue stéréotype. D'où la préférence pour la notion de stéréotype lexicalisé, affirmant d'avantage cette dynamique, que pour le simple résultat en langue que serait le stéréotype lexical. Employer une locution figurée, surtout en littérature, c'est revendiquer son stéréotype et surtout le processus de répétition qui en a fait un stéréotype.

Dans Diálogo en Re Mayor, cette répétition innervant tous les niveaux du texte, cette

\section{NOTES}

1. R. AMOSSY, A. HERSCHBERG PIERROT, Stéréotypes et clichés, Nathan, Paris, 1997, 128 p.

2. Ces deux acceptions du style ne sont pas récentes ( $C f$. note suivante) mais encore actuelles comme le montre la définition du style de Segre (1985) : «1) Conjunto de los rasgos formales que caracterizan (en su totalidad o en un momento en particular) el modo de expresarse de una persona, o el modo de escribir de un autor. 2) Conjunto de 
rasgos formales que caracteriza un grupo de obras, constituido sobre bases tipológicas o históricas » C. SEGRE, Principios de análisis del texto literario, Barcelona, Crítica, 1985. 3. «Alors qu'au XVIIIème siècle, le style était lié à une approche sociolectale, et correspondait, dans la typologie des discours, au concept sociolinguistique de registre, il devient au XIXème siècle, la caractéristique personnelle d'un écrivain et se rapproche de la conception actuelle de l'univers idiolectal » A. J. GREIMAS, J. COURTES Sémiotique: Dictionnaire raisonné de la théorie du langage, Hachette Université, Paris, 1979, p. 366.

4. O. DUCROT, T. TODOROV, Dictionnaire encyclopédique des sciences du langage, Editions du Seuil, Paris, 1972, p. 383.

5. F. LÁZARO CARRETER, « Literatura y Folklore », Estudios de lingüística, Crítica, Barcelona, 1980 p. 216 (éd. 1981). Il décrit ces deux systèmes différents de la façon suivante : « Por un lado, el carácter de co-creación y de posesión por parte de los destinatarios que hemos descrito como anejo al Folklore, no precisamente motivado por una obsesión estética ; el cual no sólo ha de ser consabido sino que además controla la conducta de los individuos en la sociedad o los hace reconocerse miembros de ella ; que adopta para estructurarse modelos cuya fertilidad ha sido demostrada, y que halla en su propia reiteración una vía segura para instalarse en la comunidad. Y por otro lado, el proceso de creación y de recepción típicamente literarios conducido por un impulso que desconoce el folklore o que no es parte fundamental de su funcionamiento : el deseo de originalidad ".

6. En France, Les Essais de Montaigne sont représentatifs de cette époque charnière. Alors que les deux premiers livres sont rythmés par des sentences et des exemples livresques, le troisième (1578-1580) marque le début de l'individualité en littérature : l'auteur devient la matière de son propre livre et l'autorité n'est plus reléguée qu'au rang d'illustration. Cf. MONTAIGNE, Les Essais (1772-1580), Quadrige/PUF, Paris, 1992 (3 volumes).

7. Au XVIème siècle, le folklore a été considéré par les auteurs comme un carcan autant formel qu'idéologique. En France, certains auteurs ont protesté contre les proverbes populaires (Adrien de Montluc, Vaugelas, Molière). En Espagne, Quevedo a opté pour une attitude plus ironique envers ces formules de savoir populaire. Cf. F. LÁZARO CARRETER (1980), O.C. p. 213.

8. J. TOMEO, Diálogo en Re Mayor, Plaza \& Janés Editores, Barcelona, 1993.

9. Ch. BALLY, Traité de stylistique française (1909), Librairie Georg \& Cie, Genève et Librairie

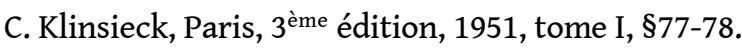

10. B. POTTIER, Linguistique générale. Théorie et description, Klinsieck, Paris, 1974, \$290.

11. G. MISRI, « Approches du figement linguistique : critères et tendances », La linguistique , vol. 23, fasc. 2, 1987, p. 72.

12. J.-L. DUFAYS, « Rumeur et stéréotypie. L'étrange séduction de l'inoriginé », Protée, vol. 32, n³, hiver 2004, pp.25-31.

13. J. L. DUFAYS, «Stéréotypes et didactique du français. Histoire et état d'une problématique », Études de linguistique appliquée, 107, 1997, pp. 315-328.

14. Cf. J.-L. DUFAYS (2004), O.C.

15..Les images sont souvent différentes d'une langue à l'autre, d'où la difficulté de traduire ces locutions.

16. Cf. à ce sujet A. IGLESIAS OVEJERO, "Elaboración de un Diccionario Histórico de Antropónimos Tradicionales en Español". ¿Es la "oralidad" un criterio? El ejemplo de Ana", Cahiers du PROHEMIO, n¹. Actes de la journée d'étude du 15 novembre 1995, Oralité, Histoire, Ecriture : réalisation et perspectives, sous la direction d'Angel Iglesias Ovejero, pp. 151-152 : "Históricamente existen, pues, textos para ser dichos, recitados o cantados, pero 
en teoría cualquier forma es oralizable, de igual manera que, en principio, nada se opone a que cualquier expresión oral sea literalizable. Esta peculiar traducción conlleva necesariamente cierta adaptación al nuevo vehículo, lo cual, en el primer caso consistirá en el hallazgo de una solución memorizable, y en el segundo en la búsqueda de una reelaboración personnal. Porque, en suma, lo que distingue a la cultura oral de la cultura escrita es que, frente a la perennidad relativa del texto, la primera reposa en la efímera memoria, siempre individual, aunque en el consenso del grupo le ofrezca la aureola de autoridad que define a la tradición".

17. E. COSERIU, «Introducción al estudio estructural del léxico », Principios de semántica estructural, Gredos, Madrid, 1981, p. 113 : « La «técnica del discurso » abarca las unidades léxicas y gramaticales (lexemas categoremas, morfemas) y las reglas para su modificación y combinación en la oración, es decir las « palabras " y los instrumentos y procedimientos léxicos y gramaticales. El « discurso repetido » abarca todo lo que tradicionalmente está fijado como " expresión », « giro ", « modismo ", « frase », o « locución " y cuyos elementos constitutivos no son reemplazables o re-combinables según las reglas actuales de la lengua $»$.

18. G. GENETTE, « Structuralisme et critique littéraire », Figure I, Editions du Seuil, Paris, 1966, p. 155.

19. C'est A. J. GREIMAS qui a introduit la notion d'isotopie dans l'analyse sémantique. Cf. Sémantique structurale, Paris, Larousse, 1966. Cette notion est plus vaste que celle de champ sémantique car, selon Greimas, elle recouvre toute " totalité de signification ». C'est-à-dire que les récurrences s'organisant dans un texte en réseau peuvent relever de la dénotation comme de la connotation, de la syntaxe comme des effets phonétiques. 20. Sylvie FOURNIÉ, Oralité, Ecriture, Réécriture. Une esthétique de la répétition dans l'œuvre de Javier Tomeo, thèse doctorale, Université d'Orléans, décembre 2002.

21. Ces paroles, comme de bien entendu, n'ont pas été du tout à mon goût, mais j'ai fait contre mauvaise fortune bon cœur et j'ai continué à sourire comme si de rien n'était. Je lui ai répondu que très bien, que chacun était libre de penser ce qu'il voulait, mais que malgré tout ce qu'il pouvait penser, je resterais convaincu que les instruments à vent en général et les trombones en particulier avaient leurs vertus et que si on en jouait dans les règles de l'art, ils pouvaient donner la chair de poule.

- Le son des trombones - lui ai-je dit, en essayant de soutenir sans sourciller son regard de vampire, est toujours noble et majestueux. Rendez-vous compte que c'est Wagner et pas un musicien à deux balles qui l'a introduit dans les orchestres symphoniques les plus modernes.

22. Pendant ce temps, Dagoberto me donna différentes définitions de l'amour (...). Il me dit, entre autre chose que l'amour était le crocodile dans le fleuve du désir, que les cœurs amoureux ne savent jamais où ils vont, que l'amour est aveugle et que l'amoureux c'est comme le poisson, il doit être frais.

23. D. BOYER, L'œuvre en vers de Salvador Espriu. Essai de systématique, thèse de Doctorat d'Etat, Université de Paris IV-Sorbonne, janvier 1994, p. 12.

24. Ah oui, essayons à nouveau ! - cria-t-il (...). Comme nous sommes présomptueux ! Comment aurions nous pu nous comprendre du premier coup ? Essayons à nouveau ! Nous avons commencé des mots croisés et nous ne pouvons pas laisser la moitié des cases vides!

- Que voulez-vous recommencer? - lui ai-je demandé.

- Notre dialogue - répondit-il. Recommençons-le. 
25. Juan, mon ami - me proposa Dagoberto -, cette fois, pour que les choses aillent mieux, nous allons nous accorder quelques facilités. Nous allons partir de zéro sans préjugés ni idées préconçues. Imaginez-vous que je suis un homme normal, disposé à partager de bon gré le compartiment avec un autre voyageur. Ressortez dans le couloir, rentrez à nouveau et asseyez-vous une nouvelle fois à la place où vous vous trouvez actuellement.

26. - Je crois qu'il vaut mieux que vous vous représentiez à nouveau, me suggéra-t-il.

- Très bien -, lui dis-je en m'armant de patience.

27. (...) il me dit que nous avions à nouveau échoué et que nous devions essayer le dialogue une fois de plus.

- Il vaut mieux ne pas perdre de temps - me demanda-t-il. Dites-moi à nouveau comment vous vous appelez, ce que vous faites et où vous allez.

28. A propos, savez-vous ce que s'est imaginé un sultan la première fois qu'il a vu un trombone à coulisse ? Tenez-vous bien car ça ne manque pas de sel : ce serviteur d'Allah a pensé que le musicien avalait puis, alternativement, retirait de sa bouche une partie de son instrument. Vous ne trouvez pas ça drôle?

29. Ensuite il commença à me raconter à nouveau l'histoire du sultan qui, lorsqu'il vit un trombone à coulisse pour la première fois, pensa que le musicien avalait puis, alternativement, retirait de sa bouche son instrument.

30. Dans la foulée, il me demanda si je connaissais l'histoire du sultan; je lui ai dit que oui et il voulut savoir qui me l'avait racontée.

- C'est-vous-même il y a un moment - lui ai-je rappelé.

- Comment est-ce possible - s'exclama-t-il en levant les sourcils -, alors que vous et moi venons de nous rencontrer?

31. Et savez-vous ce que s'est imaginé un sultan la première fois qu'il a vu un trombone à coulisse?

Cette fois-ci, j'ai fait mine d'être surpris et j'ai nié par de solennels mouvement de tête pour qu'il ait le plaisir de me raconter une nouvelle fois l'histoire.

Ce sultan - me dit-il, en introduisant une légère variation par rapport à la version précédente s'est imaginé que les trombones à coulisse étaient comestibles.

32. Puisqu'on en est à raconter des histoires drôles (...), je connais celle du sultan et du trombone à coulisse ; Est-ce que vous la connaissez ?

Je lui ai répondu à nouveau que non et tandis qu'il me la racontait pour la énième fois, le train se mit en marche.

33. Dagoberto continua à se faire l'avocat du diable et une fois de plus il remit sur le tapis l'histoire du sultan.

Croyez-vous qu'il y ait beaucoup d'instruments de musique qui puissent être confondus avec une sucette?

34. G. GENETTE, Figures III, Paris, Seuil, 1972, pp. 147 : "J'appelle évidemment ce récit, où les récurrences de l'énoncé ne répondent à aucune récurrence d'événements, récit répétitif".

35. R. BARTHES, "Littérature et discontinu", Essais critiques, Paris, Le Seuil, 1962, p. 185.

36. D. ARNOLD (sous la direction de), Dictionnaire encyclopédique de la musique, Paris, Lafond, 1988, tome I, p. 857.

37. Toccata et fugue en ré mineur de Jean-Sébastien Bach.

38. R. BARTHES (1962), O.C. pp. 185-186.

39. Diálogo en Re Mayor, p. 13. 


\section{RÉSUMÉS}

Cet article étudiera la littérarité des formes lexicales porteuses de stéréotypes (expressions figées et proverbes), très nombreuses dans le roman de Javier Tomeo, Diálogo en Re Mayor (1993). Une réflexion théorique sur la stéréotypie et le figement linguistique, amènera à introduire la notion de stéréotype lexicalisé, en la préférant à celle de stéréotype lexical. On expliquera que ces formes fixes imagées sont porteuses de répétitions connotées en raison du processus de répétitions diachronique qui a contribué à leur formation. On exposera le principe d'une isotopie de la répétition (regroupant ces répétitions connotées mais aussi toutes les répétitions discursives intra-textuelles) et on démontrera son existence en mettant en lumière la structure répétitive très originale de ce roman.

Este artículo estudiará la literaridad de las formas léxicas con estereótipos (expresiones fijas y refranes) muy numerosas en la novela de Javier Tomeo, Diálogo en Re Mayor (1993). Una reflexión teórica acerca de la estereotipia y de la fijación lingüística llevará a introducir la noción de estereotipo lexicalizado que es preferible a la de estereotipo léxico. Explicaremos que estas formas fijas y figuradas conllevan repeticiones connotadas gracias al proceso de repetición diacrónica responsable de su formación. Expondremos el principio de una isotopía de la repetición (que abarca las repeticiones connotadas pero también todas las repeticiones discursivas intratextuales) y demostraremos su existencia poniendo de realce la estructura repetitiva muy original de esta novela.

\section{INDEX}

Mots-clés : stéréotype lexicalisé, Tomeo, répétition, répétition connotée, isotopie, fugue, esthétique, expressions figées, locution, locutions, proverbes, refranes, rhétorique du stéréotype, intratextualité, littérature espagnole

Index chronologique : XXe siècle

\section{AUTEUR}

\section{SYLVIE FOURNIÉ-CHABOCHE}

Maître de conférences d'espagnol à l'Université d'Orléans, LLL (Laboratoire Ligérien de Linguistique ) 